\title{
Critical Evaluation of Relative Importance of Stress and Stress Gradient in Whisker Growth in Sn Coatings
}

\author{
PIYUSH JAGTAP, ${ }^{1}$ VIJAY A. SETHURAMAN,${ }^{1}$ and PRAVEEN KUMAR ${ }^{1,2}$ \\ 1.-Department of Materials Engineering, Indian Institute of Science, Bangalore 560012, India. \\ 2.—e-mail: praveenk@iisc.ac.in
}

The role of stress state and stress gradient in whisker growth in Sn coatings electrodeposited on brass is examined. The bulk stress in Sn coatings was measured using a laser-optics-based curvature setup, whereas glancing angle $\mathrm{x}$-ray diffraction was employed to quantify the stress near the surface; this also allowed studying the role of the out-of-plane stress gradient in whisker growth. Both bulk stress and near-surface stress in the Sn coating evolved with time, wherein both were compressive immediately after the deposition, and thereafter while the bulk stress monotonically became more compressive and subsequently saturated with aging at room temperature, the stress near the surface of the Sn coating continually became more tensile with aging. These opposing evolutionary behaviors of bulk and near-surface stresses readily reveals establishment of a negative out-of-plane stress gradient, which is required for the spontaneous growth of whiskers. The importance of the outof-plane stress gradient was also validated by externally imposing widely different stress states and stress gradients in Sn coatings using a 3-point bending apparatus. Additional whisker growth occurred in the coatings subjected to external tensile stress; however, this was accompanied by a higher negative out-of-plane stress gradient. The results conclusively demonstrate the important role of the negative out-of-plane stress gradient on whisker growth, as compared to only sign and magnitude of stress.

Key words: Curvature measurement, Sn whiskers, stress gradient, stress state, XRD

\section{INTRODUCTION}

Whisker growth in electrodeposited Sn coatings is a major concern in $\mathrm{Pb}$-free $\mathrm{Sn}$ coatings and solders used in various electronic applications. Sn whiskers, which are filamentous Sn structures with very high aspect ratio (e.g., 1:10000 ${ }^{1}$ ), readily causes a shortcircuit when it connects two adjacent current carrying conductors. Therefore, understanding the mechanism by which Sn whiskers grow is of great scientific and technological interest as it could facilitate development of effective strategies to mitigate such growth during the service life of electronic devices. It is now understood that

(Received November 24, 2017; accepted May 16, 2018; published online May 31, 2018) whisker growth in Sn coatings is a diffusion-controlled stress relaxation phenomenon, ${ }^{2}$ wherein $\mathrm{Sn}$ atoms are incessantly transported from the bulk of the coating to the root of whiskers. ${ }^{2-7}$ Moreover, compressive stress in $\mathrm{Sn}$ coatings on $\mathrm{Cu}$ or brass substrate, which is continuously regenerated due to the growth of the intermetallic compound (IMC) at the interface of Sn coating and substrate, is generally considered to be responsible for the growth of whiskers. $^{6,7}$ In fact, the mass transport of atoms from the highly compressed region to the relatively stress-free whisker root during whisker growth and an accelerated whisker growth rate under externally applied compressive stress were reported very early in $1954 .^{2}$ The stresses, both internal ${ }^{3,4}$ and external, ${ }^{2,5}$ and the stress gradients, both in-plane and out-of-plane, ${ }^{6}$ have been suggested to play a 
crucial role in whisker growth. Nevertheless, as explained below, a detailed study delineating the importance of the stress gradient and the nature (i.e., sign and magnitude) of the stress on Sn whiskering is still not available in the literature.

Preliminary evidence of the role played by stress in Sn whiskering came from classic x-ray diffraction (XRD) studies, ${ }^{7-13}$ which showed changes in the lattice spacing of the $\mathrm{Sn}$ coating and the $\mathrm{Cu}$ substrate such that the $\mathrm{Sn}$ and $\mathrm{Cu}$ layers were under compressive and tensile stresses, respectively. ${ }^{7}$ The compressive stress in Sn coatings was attributed to diffusion of $\mathrm{Cu}$ into $\mathrm{Sn}$ and the reaction between $\mathrm{Cu}$ and $\mathrm{Sn}$ that produced an interfacial $\mathrm{Cu}_{6} \mathrm{Sn}_{5}$ IMC layer in between the $\mathrm{Sn}$ coating and the substrate. In this context, a few interesting observations were made: (1) placing a thin $\mathrm{Ni}$ layer between the $\mathrm{Sn}$ and $\mathrm{Cu}$ substrates, which completely stopped whisker growth, prevented the Sn coating from becoming compressive, ${ }^{8}$ (2) the existence of a threshold compressive stress (e.g., $45 \mathrm{MPa}$, externally applied ${ }^{4}$ ) below which whiskers did not grow, (3) "baking" the Sn coating at $150^{\circ} \mathrm{C}$ for $1 \mathrm{~h}$, which led to prevalence of tensile stress in the Sn coating, prevents growth of whiskers even after long periods (e.g., $700 \mathrm{~h}^{12}$ ), and (4) the electrolytic baths that produce Sn coatings having more tensile stress (e.g., nonmethanesulfonic acid (MSA) as compared to MSA bath) were effective in reducing overall whisker growth. ${ }^{9}$ Therefore, it became a thumb rule to solely correlate whiskering with compressive stress in Sn coating. Nevertheless, contrary reports are also available in the literature. For example, Lal and Moyer ${ }^{11}$ used an XRD-based conventional $\operatorname{Sin}^{2} \psi$ method to quantify the stresses in Sn coatings deposited using various bath chemistries and current densities. However, they did not observe a consistent relationship between the residual stress and the whisker growth. This may be partly because the various Sn coatings had different thicknesses and crystallographic textures, factors which are known to significantly affect whiskering. ${ }^{10,11}$ However, as will be evident from the results obtained in this study, the above inconsistency may also be due to the lack of information on out-of-plane stress gradients.

The other set of studies illustrating the role of stress measured the curvature of the coating-substrate system to determine stresses in Sn layer. ${ }^{10,14-20}$ Although this method does not elucidate the mechanism of stress evolution, its simplicity enables simultaneous measurement of residual stress and the whisker density in real time. ${ }^{17,18}$ Thus, a direct correlation between the stress state and the whisker growth rate can be established, from which it has been shown that stress in Sn coatings rapidly approached the maximum compressive stress and, due to the whisker growth, the stress became tensile or saturated at a smaller compressive value. ${ }^{10,14,16-20}$ Curvature measurement techniques also showed the efficacy of post-deposition "baking" of the coating at $150^{\circ} \mathrm{C}$ for $1 \mathrm{~h}^{9}$ as well as alloying $\mathrm{Sn}$ with $\mathrm{Pb}^{14,17,18}$ towards preventing the build-up of compressive stress in the Sn coatings, eventually resulting in whisker-free Sn coatings.

Nevertheless, contradictory to the general consensus discussed above, the growth of whiskers was also observed in Sn coatings under tensile stress, including reflowed $\mathrm{Sn}$ coatings ${ }^{21,22}$ and a Sn-Mn alloy coating. ${ }^{15}$ In particular, it was observed that whisker growth in Sn-Mn alloy was observed within a few hours after the deposition and coating remained under tensile stress during the entire observation period for whisker growth. ${ }^{15}$ Furthermore, it was reported that the Sn coatings baked at $150^{\circ} \mathrm{C}$ for $1 \mathrm{~h}$ developed compressive stress after prolonged aging (e.g., 150 days or $3600 \mathrm{~h}$ ); however, these coatings still remained whisker-free. ${ }^{13}$ These "anomalous" observations suggest that the nature of stress alone may not be the sole factor driving whisker growth.

Compared to the effect of stress on whisker growth, the role of the stress gradient has received less attention. ${ }^{6,13,23,24}$ The only known experimental study $^{13}$ employing an XRD-based technique for measuring stress as a function of distance from the surface reported that a negative out-of-plane stress gradient is necessary for whisker growth. However, since x-rays have a limited depth of penetration in Sn coatings, the stress gradient could only be measured in the top $1 \mu \mathrm{m}$ of a $4 \mu \mathrm{m}$ thick coating using this method. It should be noted that growth of the IMC layer, which is attributed to the generation of compressive stress in Sn coatings showing whisker growth, occurs at the interface of Sn and the substrate, which often cannot be reached by x-rays. This has been a common shortcoming of previous studies ${ }^{8,9,12}$ using XRD techniques for the measurement of stress in Sn coatings, as the measured stress values are not representative of the entire Sn coating since the stress gradient spans the entire Sn coating. In a later report that used synchrotron micro-diffraction to study the local stress field surrounding whiskers, ${ }^{6}$ it was reported that both in-plane and out-of-plane stress gradients are essential for whisker growth.

In the context of evaluating the importance of stress, the role of external stress has also been studied in the past by several research groups $^{2,5,25-30}$; however, contradictory results were often reported. For example, a few studies reported accelerated whisker growth under applied compressive stress, ${ }^{2,5,25}$ while others reported the contrary. ${ }^{26}$ Furthermore, several studies reported that the whiskers grown under compression were longer and greater in number than they were in the stressfree region of the sample, ${ }^{27,30}$ while others reported that whisker growth was greatest in Sn coatings under tensile stress, and that whiskers in coatings under tensile stress were longer than whiskers in the compressively stressed Sn coatings. ${ }^{28,29}$ 
However, explicit explanation of the above "apparently anomalous" results or the dichotomy in the results was not provided in any of the studies.

It is evident from the aforementioned discussion on stress measurement in Sn coatings and the effect of stress and stress gradient on whisker growth that the role of stress in whisker growth still remains controversial. In particular, the growth of whiskers under tensile stress is "anomalous" to the general consensus and has never been systematically addressed in previous works. In addition, the above discussion indicates that experimental evidence confirming (or refuting) the importance of the stress gradient and the nature of the stress (i.e., compressive or tensile as well as magnitude) on whisker formation remains limited. Accordingly, this study aims to critically examine the relative importance of the nature of the stress and the stress gradient on Sn whiskering by quantifying the evolution of residual stresses across the thickness of Sn coatings during aging and by imposing widely different external stresses and stress gradients on Sn coatings. Thus, this study should contribute to a fundamental understanding of whisker growth. The results and analysis shown here suggest that whisker growth depends primarily on the stress gradient, and hence tracking the prevalent stress gradient in the coating may resolve the dichotomy in the results reported by various groups.

\section{EXPERIMENTAL MATERIALS AND PROCEDURE}

\section{Estimation of Stress Using Substrate Curvature Measurement}

The curvature of the substrate was recorded using a setup based on laser optics. Using laseroptics to measure curvature requires preparation of a substrate with flat and reflective surfaces. This can be achieved via the following procedure. Brass coupons of size $2 \times 2 \mathrm{~cm}^{2}$ and a thickness of $300 \mu \mathrm{m}$ were cut from a large sheet using wire electrodischarge machining (W-EDM). Both surfaces of the brass coupons were then metallographically polished up to a $0.05 \mu \mathrm{m}$ surface finish. Subsequently, a coupon of $1 \times 1.5 \mathrm{~cm}^{2}$ size was sectioned from the central region of the polished coupon using W-EDM at slow speed. This produced the reasonably flat and highly reflective surface required for measuring stress in the Sn coating using an optical technique for measuring wafer curvature.

For a detailed description of the experimental setup, one can refer to, ${ }^{31-33}$ as only the important aspects are described here for the sake of continuity. The wafer curvature measurement setup was placed on a vibration isolation optical table to preclude error due to vibrations over very long periods of observation. The setup comprised a fibercoupled laser source, a beam splitter, an optically flat mirror and a charge-coupled device (CCD) detector connected to a computer that allowed continuous monitoring of the substrate curvature. The inclination of the beam splitter, which was placed in the path of the incident laser beam, relative to the laser beam was controlled to obtain an array of multiple laser spots, and the spacing between the laser spots after being reflected by the substrate or wafer was continuously monitored in real time using the CCD detector. The curvature, $\kappa$, was calculated by measuring the fractional change in the laser spot spacing, and the film stress, $\sigma_{\text {f }}$, was subsequently estimated using Stoney's equation as follows:

$$
\sigma_{\mathrm{f}}^{\prime}=\frac{E_{\mathrm{s}}}{6\left(1-v_{\mathrm{s}}\right)} \frac{d_{\mathrm{s}}^{2} \kappa}{d_{\mathrm{f}}}
$$

where $E_{\mathrm{s}}$ and $v_{\mathrm{s}}$ are the Young's modulus and Poisson's ratio of the substrate, respectively, and $d_{\mathrm{s}}$ and $d_{\mathrm{f}}$ are thicknesses of the substrate and the film, respectively. However, it should be noted that Stoney's formula, as given in Eq. 1, is strictly valid only if the substrate is absolutely flat prior to the film deposition, which is often not the case. Therefore, change in the curvature $(\Delta \kappa)$ was calculated from the curvature of the substrate before and after the electrodeposition of the Sn coating. This necessitated that the value of the curvature, $\kappa$, in Stoney's equation, as given in Eq. 1, should be replaced by change in curvature, $\Delta \kappa$. This allowed measurement of a change in the stress in the film. It should be noted that the IMC layer formed between $\mathrm{Sn}$ and brass was discontinuous and hence, as will be discussed later, the change in curvature due to the discontinuous IMC layer would be negligible. ${ }^{34,35}$ Therefore, the effect of the IMC layer on the stress in Sn coating was neglected.

After measuring the initial curvature of the polished brass coupons, they were coated with a 4 $\mu \mathrm{m}$ thick layer of Sn using electrodeposition via an acidic stannous sulfate bath. Prior to electrodeposition, brass coupons were cleaned using isopropyl alcohol followed by an acid rinse to remove the surface oxide layer. Pure Sn (99.99\% purity) was used as the anode while brass coupons were attached to the cathode of an electrolytic cell. The electrolytic solution was stirred using a magnetic needle at 200 rotations per minute to maintain uniform hydrodynamic conditions. The electrodeposition was performed at a constant bath temperature of $40^{\circ} \mathrm{C}$ using a current density of $20 \mathrm{~mA} / \mathrm{cm}^{2}$ for $330 \mathrm{~s}$. Sn was selectively deposited on only one side of the brass coupons by masking the other side using Kapton ${ }^{\circledR}$ tape. Following electrodeposition, the Kapton ${ }^{\circledR}$ tape was removed by dipping the samples in an acetone bath. The change in the curvature of the substrate was then continuously monitored in real time using the laser-optics-based system. It should be noted that the laser reflected from the backside of the brass coupon, which was not coated with Sn. 


\section{Stress Measurement Using X-ray Diffraction}

The stress in the vicinity of the surface of the Sn coating was measured using another set of representative samples, deposited at the same bath temperature and current density mentioned in the "Estimation of Stress Using Substrate Curvature Measurement" section, using an x-ray diffractometer (Bruker D8 Discover TXS) with a parallel beam geometry. The turbo x-ray source (TXS) rotating anode produced the highest intensity x-rays, which allowed measurement of the shift in high-angle peaks that is not captured by an ordinary x-ray diffractometer operating at lower x-ray currents. $\mathrm{CuK} \alpha$ radiation from a rotating anode operating at $45 \mathrm{kV}$ and $100 \mathrm{~mA}$ was used in this study. A $0.2 \mathrm{~mm}$ incident slit was used to restrict the beam size of $x$ rays. The sample was mounted on a Eulerian cradle, which allowed for sample tilts and rotation. The stress measurement was performed in $\omega$-mode (or glancing angle mode), ${ }^{36}$ which allowed measurement of the inter-planar spacing of the set of $(h k l)$ planes that are inclined towards the sample surface. The angle of incidence, $\alpha$, was varied from $1^{\circ}$ to $10^{\circ}$, thus changing the effective penetration depth of the $\mathrm{x}$-rays. The penetration depth of the $\mathrm{x}$-ray beam, $\tau$, can be calculated from the incident angle $(\alpha)$ and the exit angle $(\beta)$, as follows ${ }^{37}$ :

$$
\tau=\frac{\sin \alpha \sin \beta}{\mu(\sin \alpha+\sin \beta)}
$$

where $\mu$ is the mass absorption coefficient ( $\mu / \rho$ equal to $274 \mathrm{~cm}^{-1}$ for $\mathrm{Sn},{ }^{38}$ where $\rho$ is the density of $\mathrm{Sn}$ ). Thus, the variation in incidence angle enabled probing of strain in (501)-planes at different penetration depths, ranging from 0.1 to $0.76 \mu \mathrm{m}$ in a 4 $\mu \mathrm{m}$ thick coating, from the free surface of the Sn coatings. Therefore, the stress was measured up to a maximum penetration depth of only $\sim 15 \%$ of the total thickness of the coating and hence reasonably represented average stress in the top layer of the coating or near-surface stress.

As mentioned above, the diffraction peak corresponding to the (501)-reflection of Sn was collected at various $\alpha$ and a shift in the position of peak was monitored. The peak measurement was performed with a step size of 0.0005 and a step time of $3 \mathrm{~s}$. Therefore, measurement of one peak took approximately $\approx 2 \mathrm{~h}$ and the entire measurement took approximately $\approx 20 \mathrm{~h}$. The peak positions of the (501)-reflection at different $\alpha$ were calculated by a peak fitting procedure in the Origin ${ }^{\circledR}$ software using the Pearson VII peak function. ${ }^{37}$ The average stress was calculated from the linear fit between $\varepsilon_{h k l}^{\psi}$ (which is $\psi \varphi$-component of the strain corresponding to ( $h k l$ )-plane) and $\sin ^{2} \psi$, as dictated by the following relationship between the strain measured using XRD and the mechanical stress tensor for the condition of a macroscopically isotropic specimen under rotationally symmetric plane stress (i.e., $\sigma_{11}=\sigma_{22}=\sigma_{\mathrm{f}}$ and $\sigma_{12}, \sigma_{13}, \sigma_{23}$ and $\sigma_{33}=0$ ):

$$
\varepsilon_{h k l}^{\psi \varphi}=\left(\frac{1}{2} S_{2}^{h k l} \sin ^{2} \psi+2 S_{1}^{h k l}\right) \sigma_{\mathrm{f}}
$$

where $S_{1}^{h k l}$ and $S_{2}^{h k l}$ are diffraction elastic stiffness constants. The strain-free lattice spacing of the (501)-reflection provided by the Joint Committee on Powder Diffraction Standards (JCPDS) was used to calculate the strain using the following equation.

$$
\varepsilon_{h k l}^{\psi \varphi}=\frac{d_{h k l}-d_{0}}{d_{0}}
$$

\section{Application of External Stress and Stress Gradient Using 3-Point Bending}

To study the effects of the external stress and stress gradient on whisker growth, a 3-point bending setup was used. First, Sn coatings were electrodeposited, using the process described earlier in the "Estimation of Stress Using Substrate Curvature Measurement" section with the same bath temperature and current density, on only one side of a $200 \mu \mathrm{m}$ thick and $5 \mathrm{~cm}$ long brass strip. Figure 1 schematically illustrates the strategies employed for application of external compressive and tensile stresses. The advantages of such a setup are: (1) it allowed the imposed stress and stress gradients to be quantified using simple finite element analysis (FEA) of a bimetallic strip under bending load, (2) tensile and compressive stress of the same magnitude can be imposed in the coatings on the opposite sides of the sample at the same time, as shown schematically in Fig. 1, (3) data corresponding to various tensile and compressive stresses can be obtained using one experiment, and (4) bending of the Sn coating in such a manner also produced stress gradients along the thickness of the coating (and substrate). In particular, as shown in Fig. 1b, the application of stress in such a way created the stress gradients along the thickness direction, such that the surface of the Sn coating was at maximum tension or compression, depending on the configuration chosen in Fig. 1a.

FEA of the bimetallic strip with geometry similar to that of the actual sample was also employed using ANSYS $^{\circledR}$, a commercial FEA software package, for quantification of the stress state as well as the stress gradient across the coating thickness imposed by the 3-point bending. FEA was performed by assigning the time independent elastic-plastic property for Sn. An experimental stress-strain curve of pure Sn obtained at a constant strain rate of $10^{-3}$ $\mathrm{s}^{-1}$ was used for the Sn coating (and hence we do not assume ideal plasticity for Sn in our simulations). The brass substrate was assumed to be elastic, which is reasonable for a thick brass substrate.

After electrodeposition and loading of the sample into a 3-point bending fixture, it was stored in a 

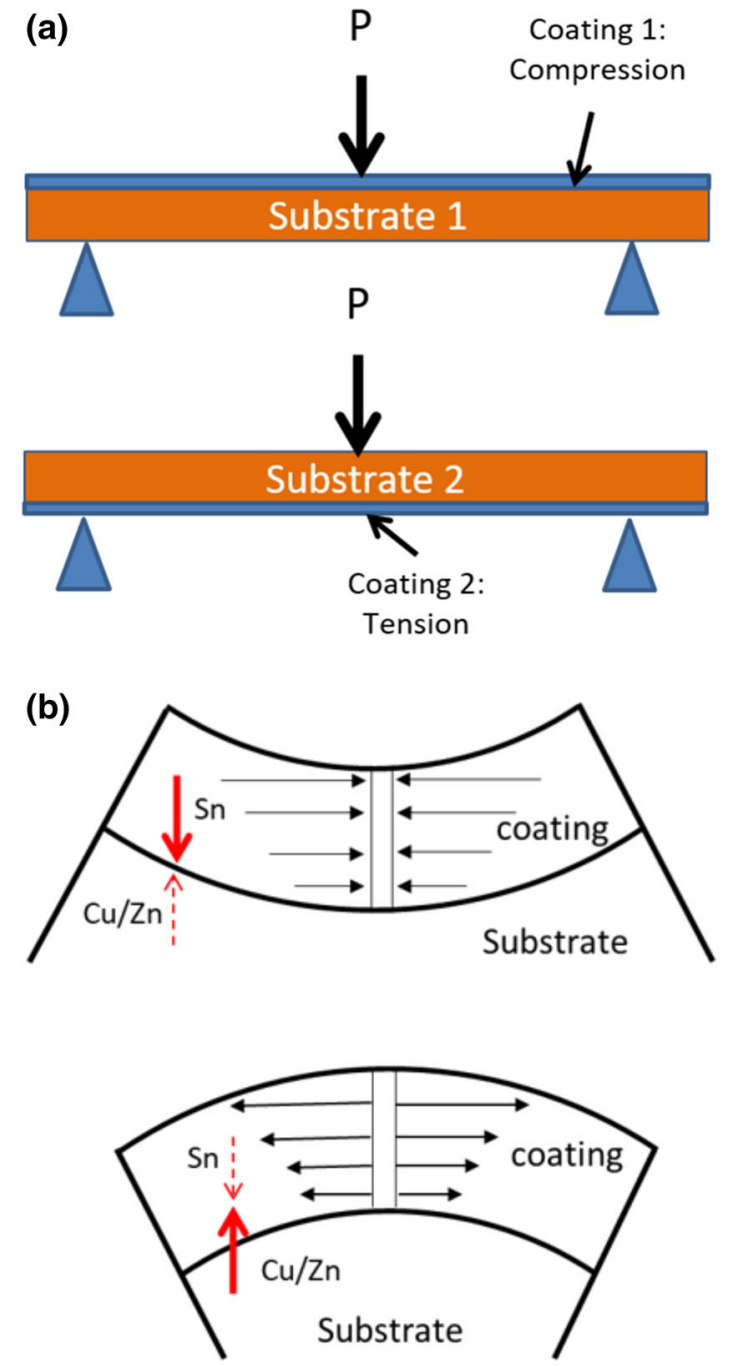

Fig. 1. (a) 3-Point bending schemes used to apply external stress and stress gradient on the Sn-coated brass. (b) Schematic illustration of the stress gradient produced as per the schematic shown in (a). The size and the direction of horizontal arrows in (b) denote the magnitude and sign of the dominant stress component in the $\mathrm{Sn}$ coating, whereas the thick and broken vertical arrows show the direction of significant and less-significant atomic transport, respectively. The vertical channel (onto which the stresses are shown acting) in (b) represent a columnar grain of Sn coating.

hot air oven at $50^{\circ} \mathrm{C}$ and whisker density as well as the $\mathrm{Cu}_{6} \mathrm{Sn}_{5}$ IMC layer was observed after 7 days. The IMC layer was observed by cross-section imaging and chemical etching of the Sn coatings. The sample for cross-section examination was prepared using dual beam focused ion beam (FIB) machining, while the Sn coating was etched away using a warm solution of 7 parts o-nitro-phenol and 1 part $\mathrm{NaOH}$. Whisker density in the samples was evaluated at several distances from the center (i.e., maximum deflection point) and correlated with the magnitude of the stress and stress gradient that exist at the particular location or corresponding region.

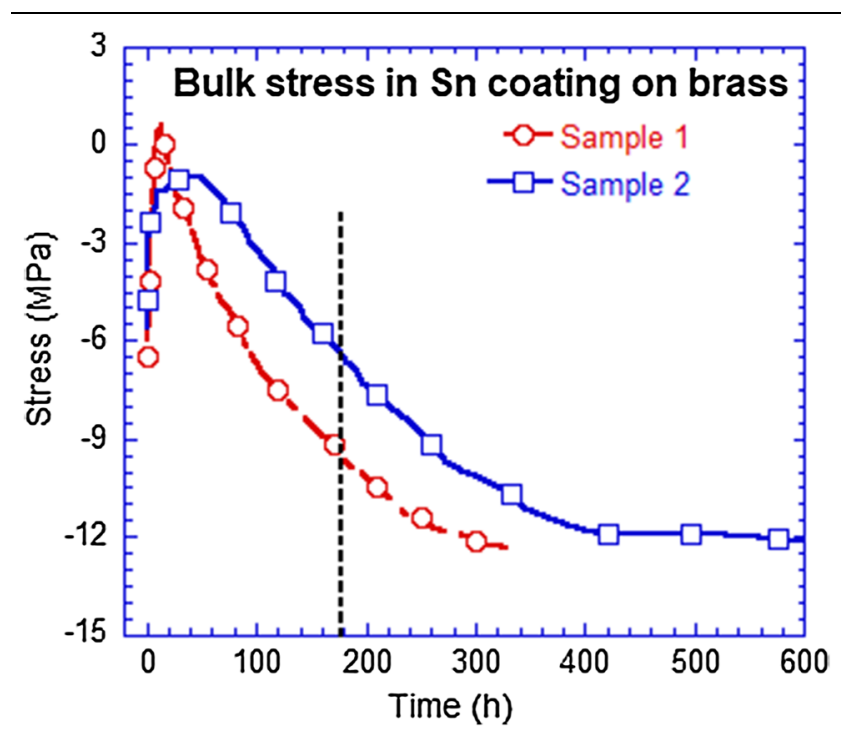

Fig. 2. Bulk stress evolution over time in two $4 \mu \mathrm{m}$ thick Sn coatings electrodeposited on a $200 \mu \mathrm{m}$ thick brass substrate, as measured using the laser-optics-based curvature measurement setup. The vertical line shows the approximate time at which the first set of whiskers started to grow in these coatings. Samples were stored under ambient conditions.

\section{RESULTS}

\section{Substrate Curvature Measurements}

Figure 2 shows the evolution of bulk stress in a representative Sn coating aged at room temperature, as estimated using Eq. 1. Careful observation of Fig. 2 readily reveals the following: (1) the residual stress in the Sn coating immediately after deposition was compressive, (2) the initial compressive stress in the Sn coatings relaxed rapidly during the initial hours of aging following the deposition, (3) thereafter, stress became progressively more compressive with aging, and (4) the compressive stress showed saturation near - $12 \mathrm{MPa}$ (which is close to the yield strength of Sn) after $400 \mathrm{~h}$ of aging. Interestingly, whiskers were observed in this sample after just 7 days (i.e., $\sim 180 \mathrm{~h}$ ), suggesting that whiskering did not completely stop the buildup of stress in Sn coatings. However, saturation in stress or the establishment of a steady-state value for stress after $400 \mathrm{~h}$ suggests that once a very large number of whiskers had nucleated, the stress generated due to IMC formation at the substratecoating interface and the stress relaxation due to whisker growth balanced each other. It should be noted that the measured stress corresponds to the entire Sn coating (i.e., both bulk and surface). However, it is expected that the average value will be affected more by the volume of the material away from the top surface as compared to the surface and hence the stress shown in Fig. 2 can be assumed to represent the bulk stress. 


\section{Stress Measurement Using X-ray Diffraction}

Figure 3a shows the peak profile of the (501)planes obtained at different angles of incidence. The vertical arrow in Fig. 3a shows the expected position of the peak of the (501)-reflection in an unstrained crystal, and any deviation from this arrow can be attributed to the strain in the sample. Figure 3 clearly shows a significant shift in the peak position of the (501)-reflection of the Sn coating after 5 days of aging. Subsequently, the strain values at different incidence angles were plotted against $\sin ^{2} \psi$ to calculate the average stress in the top $0.76 \mu \mathrm{m}$ of the coating. As directed by Eq. 3, the strain values at different incidence angles, $\omega$, and therefore at different $\psi$ tilts, were plotted as function of $\sin ^{2} \psi$ (see Fig. 3b). As shown in Fig. 3b, a reasonably linear fit between the strain and $\sin ^{2} \psi$ values was obtained. The stress was calculated from the slope of the linear fit using Eq. 3. It should be categorically noted that the value of stress reported following this method is the average stress near the surface of Sn coatings (i.e., top $0.7 \mu \mathrm{m}$ of the coating) and does not represent the bulk stress. Consequently, stress near the surface region was measured using the aforementioned technique after different aging times of up to 30 days after electrodeposition.

Figure 4 shows the $\operatorname{Sin}^{2} \psi$ plot of the same coating, whose data is shown in Fig. 3, as recorded at different times along with the variation of stress with time. It can be readily observed from Fig. 4 that the stress in the as-deposited samples was compressive and became less compressive (i.e., more tensile) with aging time. This suggests that whisker growth is a surface relief process, such that surface stress becomes continuously less compressive during whisker growth. A comparison of Figs. 2 and $4 \mathrm{~b}$ clearly suggests that the stress gradient in the Sn coating between the bulk and the top layer of the coating became more negative (i.e., negatively increased) with increased storage time. The implication of this observation on whisker growth will be discussed later.

It should be noted that the effects of the crystallographic texture and grain interaction between polycrystalline grains and the constraints imposed on neighboring grains were not considered in this XRD stress analysis. The analysis assumes rotationally symmetric equal biaxial stress in Sn and a macroscopically isotropic specimen. Furthermore, as whiskers start to a grow at a particular location, the stress state in the vicinity of the whisker grain will change. Such local variation in the stress field was not captured using the XRD (as well as curvature measurement) analysis performed herein. Nevertheless, it does capture the evolution of the average (or global) near-surface stress in the Sn coating as a function of aging time.
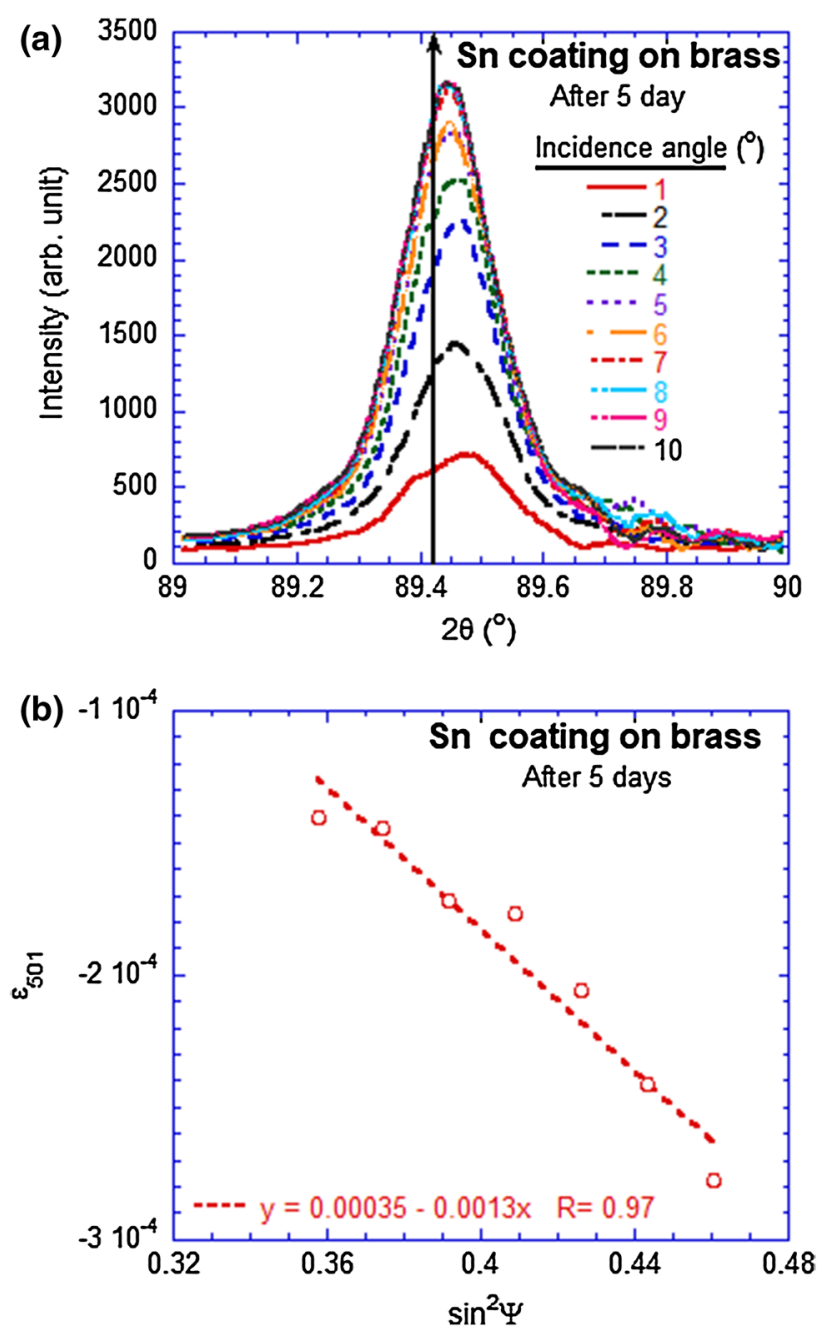

Fig. 3. (a) Peak profile of the (501)-reflections of the Sn coating obtained at different incidence angles, $\omega$, shown in the legend. The data was collected 5 days after the electrodeposition. (b) Strain versus $\sin ^{2} \psi$ plot for the Sn coating deposited on brass as recorded after 5 days after electrodeposition. The legend shows the equation obtained from the best linear curve fit, along with the curve fitting parameter, $R$. The sample was stored under ambient condition.

\section{Effect of External Stress on Whisker Growth}

As discussed in the "Application of External Stress and Stress Gradient Using 3-Point Bending" section, an external stress along with a stress gradient was imposed on the Sn-coated brass strips using a 3-point bending setup. The stress variation in the Sn-coated brass strip at various distances from the center (i.e., point of maximum deflection) is shown in Fig. 5. If the $\mathrm{Sn}$ is allowed to deform plastically with negligible hardening, a saturation in stress can be observed. Henceforth, the stress values, at different locations along the length of the Sn-coated strip reported in this study, are the values "predicted" using the FEA model incorporating plasticity. It is noteworthy that the stresses mentioned in Fig. 5 occur at the instant of loading 

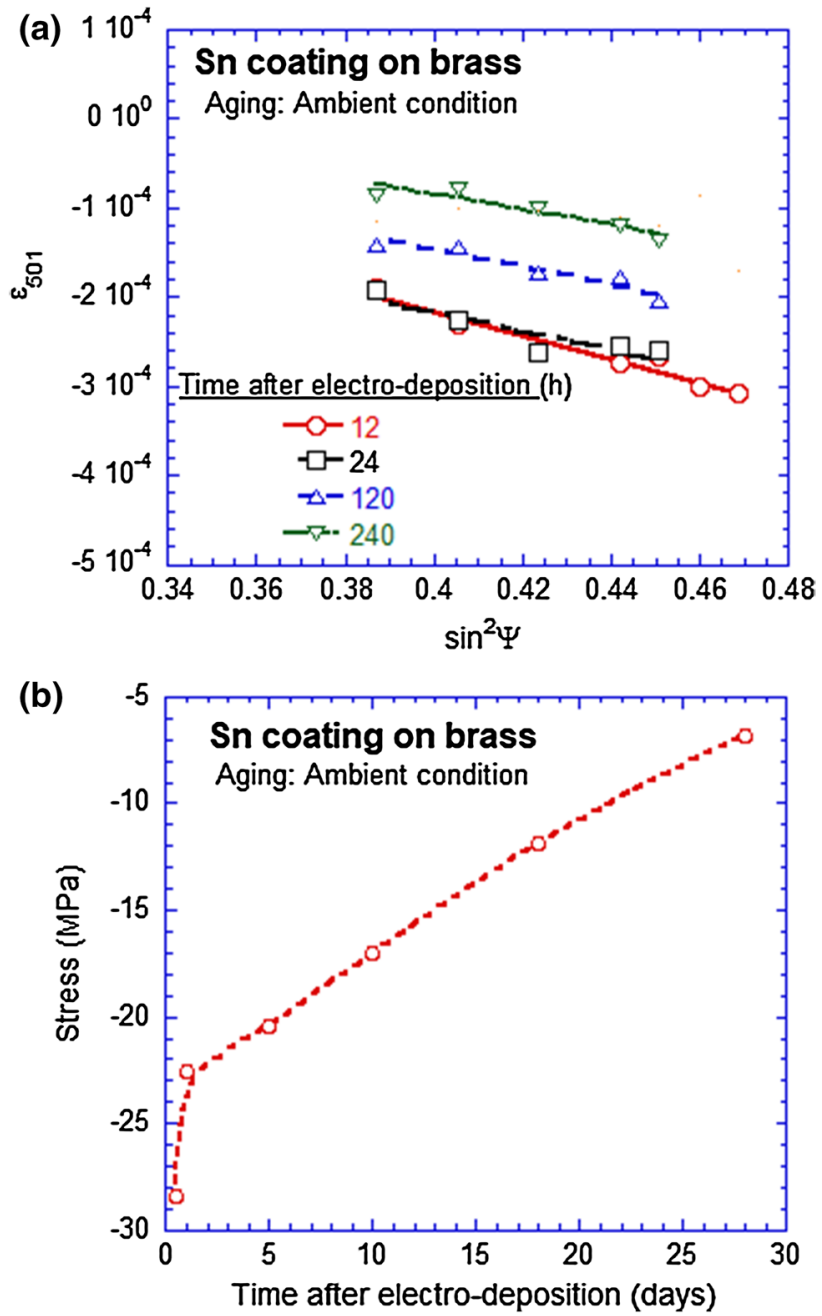

Fig. 4. (a) A few $\operatorname{Sin}^{2} \psi$ plots of the Sn coating on brass substrate obtained at various time points following electrodeposition, and (b) evolution of the average surface stress with aging time following electrodeposition.

and are therefore based on time-independent plasticity in Sn. It is possible that the power-law creep may relax the stresses in the Sn layer significantly. However, as will be discussed later, stress relaxation due to creep remains insignificant compared to the stress build-up due to the IMC growth and the constraining effect of the oxide layer on the free surface.

Figure 6 shows a few representative micrographs of the $\mathrm{Sn}$ coatings stored at $50^{\circ} \mathrm{C}$ for 7 days under applied tensile and compressive stresses of similar magnitude. It is evident from Fig. 6 that the whisker density was much higher in the Sn coating under tensile stress. Moreover, in addition to the higher density of whiskers in the Sn coatings under tensile stress, the average length of the whiskers was significantly higher than those of Sn coatings loaded under compressive stress of equal magnitude.

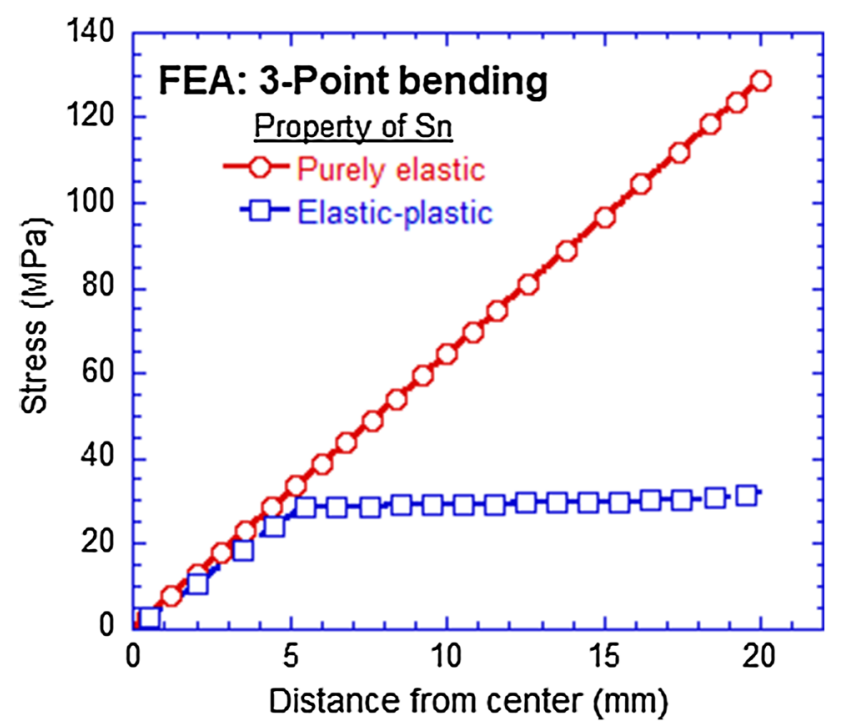

Fig. 5. Variation of magnitude of the stress in the Sn coating, subjected to 3-point bending, as a function of distance from the center (or point of maximum deflection), as predicted by FEA. The stress values in $S n$ vary linearly and become very high if $S n$ is not assumed to be an elastic-plastic material.

Figure 7 shows whisker density, measured as number of whiskers per unit area, as a function of the magnitude of the stress and the stress gradient. Whisker density was calculated at different fixed nominal distances (e.g., $0 \mathrm{~mm}, 5 \mathrm{~mm}, 10 \mathrm{~mm}$, $15 \mathrm{~mm}, 20 \mathrm{~mm}$ and $22.5 \mathrm{~mm}$ ) from the center. A region of $0.8 \mathrm{~mm}$ length such that the region lies $\pm 0.4 \mathrm{~mm}$ on each side of the nominal distance mentioned above was observed for whisker density calculation. Furthermore, the whisker density values reported in Fig. 7 are the average whisker density over the region mentioned above, and the stress as well as stress gradient shown in Fig. 7 correspond to the FEA predicted value at the center of the $0.8 \mathrm{~mm}$ wide region wherein the whisker density was measured. It is evident from Fig. 7a that, consistent with Fig. 6, the whisker growth was very rapid when the Sn coating was subjected to tensile stress. In addition, the whisker density increased monotonically with the tensile stress and decreased slightly with an increase in the magnitude of the compressive stress. Interestingly, the effect of increasing tensile stress on whisker density was significantly more prominent than that of increasing compressive stress. Similarly, Fig. 7b shows that whisker density monotonically increased with the magnitude of the negative stress gradient across the thickness of Sn coating imposed due to bending.

It should be noted that the externally applied stress (and hence stress gradient across the coating thickness due to bending) might also affect the diffusion of $\mathrm{Cu}$ and $\mathrm{Zn}$ atoms from the substrate into the Sn coating ${ }^{39}$ and hence the growth kinetics of the interfacial IMC layer in the coatings within 

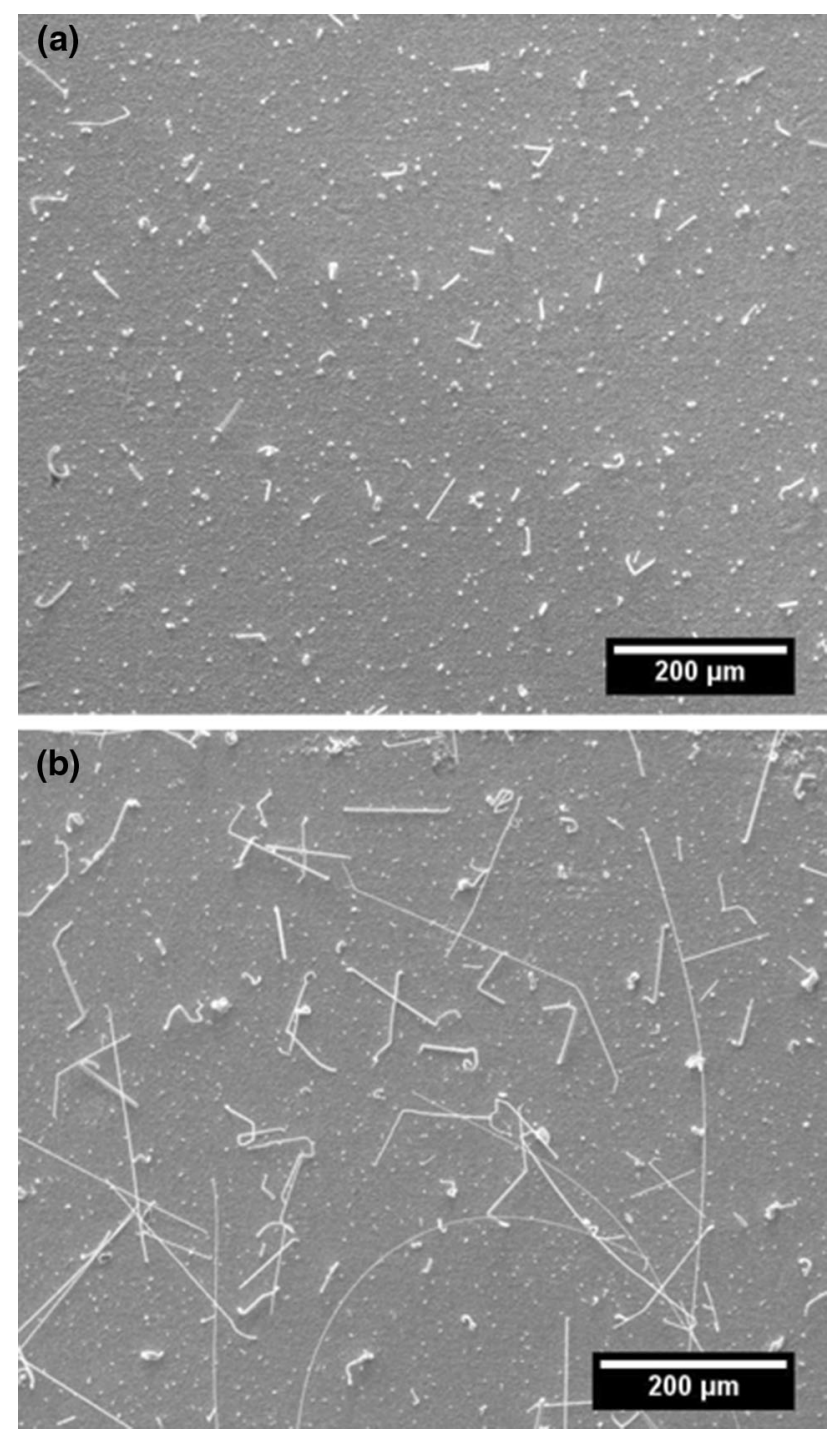

Fig. 6. Representative micrographs, obtained using scanning electron microscope, of Sn coatings subjected to external (a) compressive and (b) tensile stresses of similar magnitudes. The stresses were applied using a 3-point bending setup.

compressive and tensile regions is expected to be significantly different. Therefore, the IMC growth in the samples loaded using the 3-point bending setup was monitored. Figure 8 shows micrographs revealing the cross-section of the Sn coatings under compressive and tensile stresses of similar magnitude. Careful observation of Fig. 8 reveals that the thickness of the interfacial IMC layer in the Sn coatings under tensile stress was $1.6 \pm 0.4 \mu \mathrm{m}$, while it was $1.5 \pm 0.4 \mu \mathrm{m}$ in the coatings under compressive stress of similar magnitude. Therefore, the IMC thickness, as calculated using analysis of the cross-sectional micrographs, statistically did not show noticeable dependence on the sign of the stress or stress gradient.

Figure 9 shows the top view of the interfacial IMC layer as obtained after selective etching of the Sn
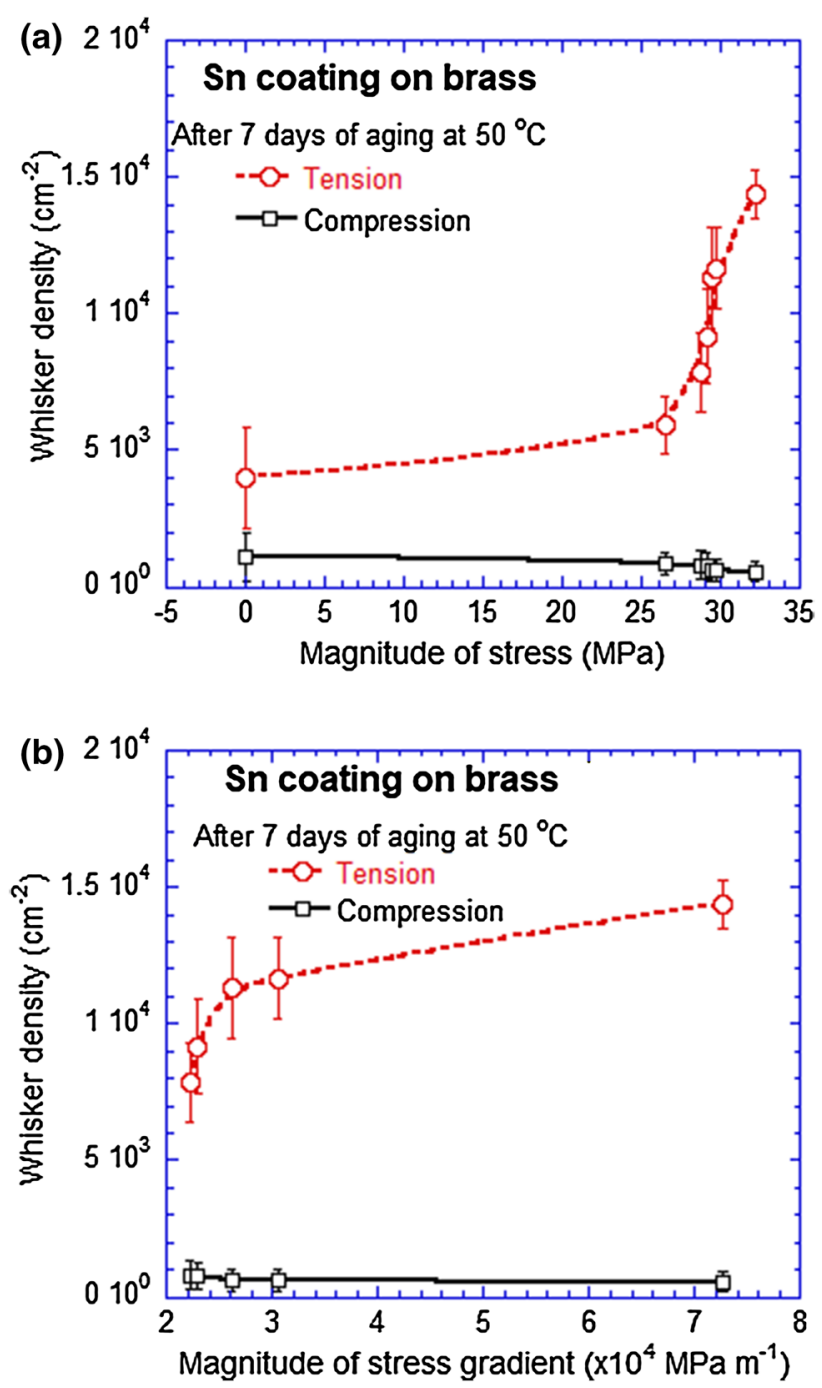

Fig. 7. Whisker density observed in the samples loaded using a 3point bending setup at $50^{\circ} \mathrm{C}$ after 7 days of electrodeposition as function of the magnitude of the (a) stress and (b) stress gradient across the thickness of the Sn coating.

coating. Figure 9 clearly shows that the IMC formed on the tensile side of the coating (substrate) possessed significantly greater density than the IMC layer formed on the side of compression. The area fractions of IMC, calculated using image analysis, on the tensile side and the compressive side were 0.65 and 0.43 , respectively. Therefore, it can be inferred that although the sign of the stress in the sample loaded under 3-point bending did not affect the overall thickness of the IMC layer, the overall volume of IMC was drastically affected by the nature of the stress, as larger tensile stress led (along with the negative out-of-plane stress gradient) to an increase in the volume of the interfacial IMC layer. Figure 9 also reveals that the IMC layer grown under externally applied stress retained its scallop-like nature. 
Critical Evaluation of Relative Importance of Stress and Stress Gradient in Whisker Growth in Sn Coatings
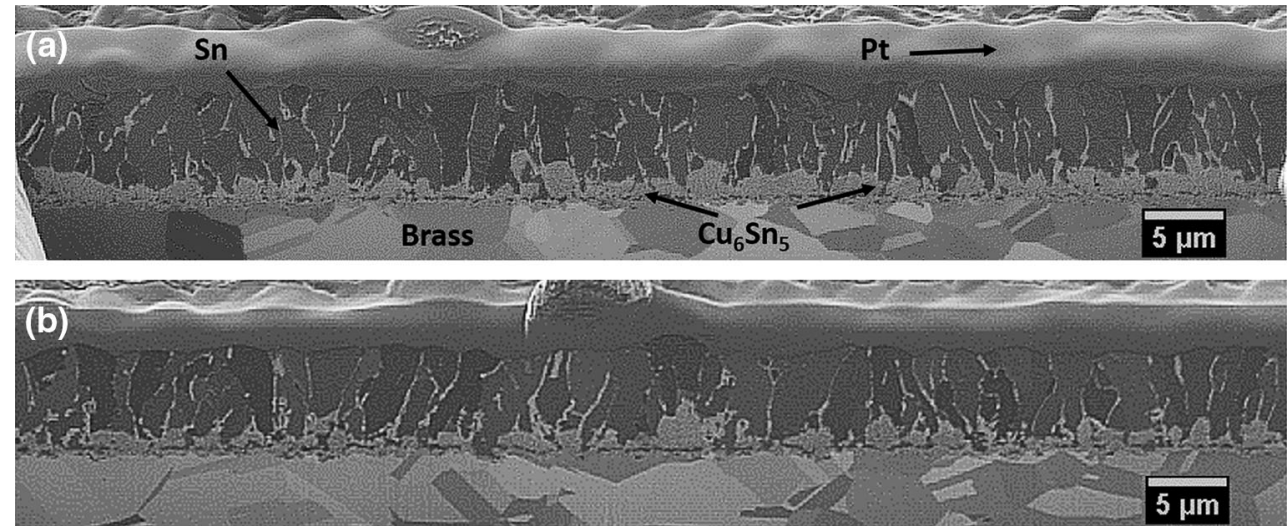

Fig. 8. Representative micrographs showing the cross-section of Sn coatings subjected to (a) tensile and (b) compressive stresses of similar magnitude.
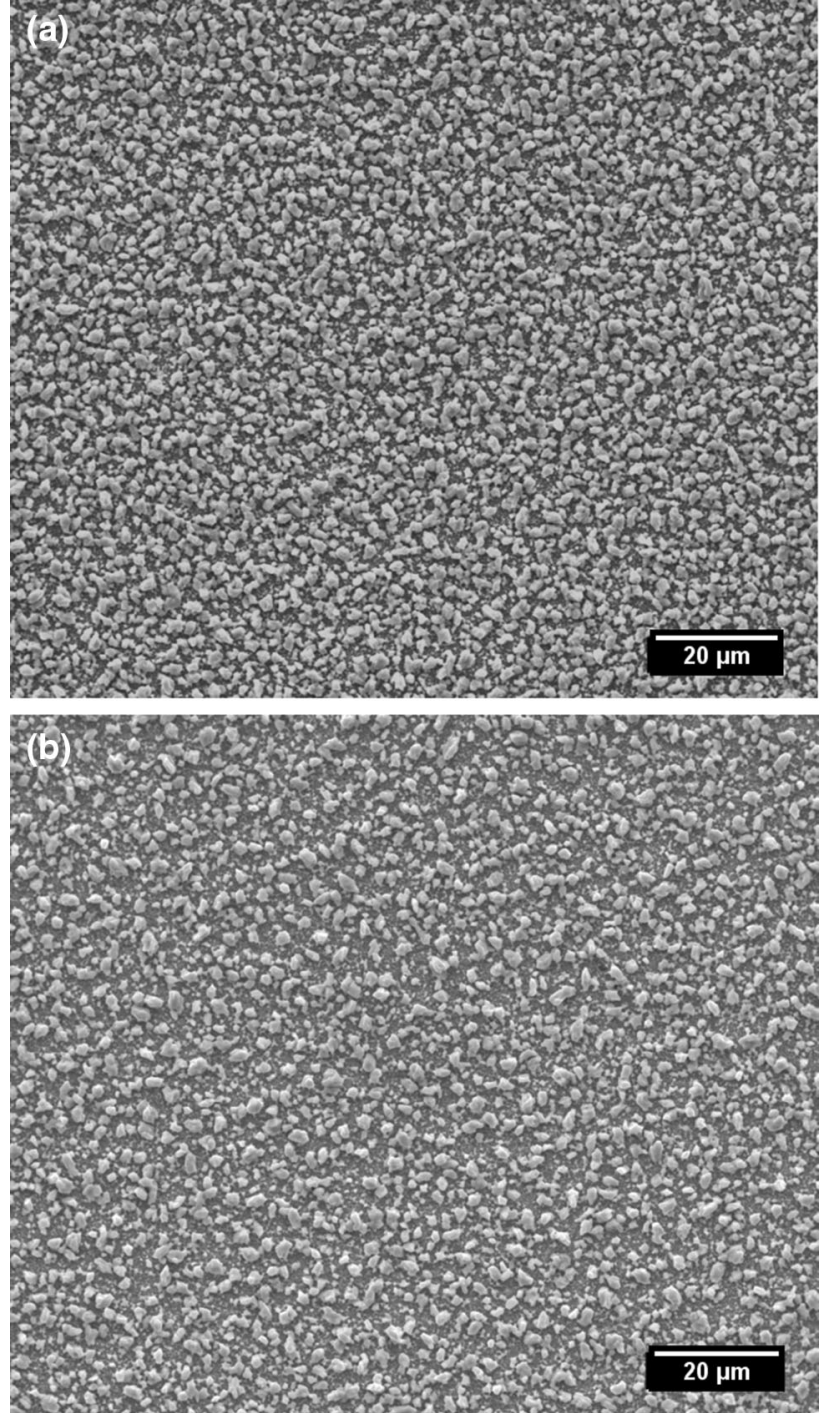

Fig. 9. Top view of the IMC layer after selectively removing the Sn coatings exposed to (a) tensile and (b) compressive stresses of similar magnitudes.

\section{DISCUSSION}

\section{Effects of Stress and Stress Gradient on Whisker Growth}

The initial residual bulk stress in the deposited Sn coatings was compressive (see Fig. 2). Assuming the stress in Sn coatings due to growth of the IMC layer is insignificant immediately after electrodeposition, the initial residual stress in the coating may originate from mismatch in the coefficients of thermal expansion (CTE) of Sn and brass or it may be inherent to the electrodeposition process. It should be noted that Sn has higher CTE compared to the brass substrate. The residual stress in Sn due to CTE mismatch can be estimated using the following simplified (1-D) equation:

$$
\sigma_{\mathrm{Sn}}=M \Delta \alpha \Delta T
$$

where $M$ is equal to $E /(1-v)$, where $E$ and $v$ are the Young's modulus and Poisson's ratio of Sn, respectively, and $\Delta \alpha$ is the difference in CTE of Sn and brass. Now, taking the elastic modulus of $50 \mathrm{GPa}$ for $\mathrm{Sn},{ }^{40}$ the residual stress in Sn due to the temperature difference between the electrodeposition bath temperature and the ambient temperature will be 5.2 $\mathrm{MPa}$ (i.e., tensile). Therefore, the residual compressive stress in the Sn coatings on brass must be inherent to the electrodeposition process (i.e., due to compressive stress developed in the pre-coalescence regime of film growth or the insertion of extra atoms at the grain boundary in the post-coalescence regime).

It is important to note that the power-law creep and other recovery processes will also aid in the relaxation of the built-in compressive stress during deposition, especially when a significant volume of the IMC and surface oxide has not formed (i.e., during and immediately after deposition). This is expected because the ambient temperature $\left(\sim 25^{\circ} \mathrm{C}\right)$ is very high for $\mathrm{Sn}\left(\sim 0.58 T_{\mathrm{m}}\right.$, where $T_{\mathrm{m}}$ is the melting temperature). However, with continued 
aging at room temperature, the columnar grain boundaries of Sn would become pinned at the top and bottom due to the oxide layer and IMC layer formed at the grain boundaries terminating at the free surface and the substrate, respectively. The formation of the oxide on the free surface of Sn prevents efficient stress relaxation via the creep processes. Therefore, the build-up of compressive stress due to IMC growth dominates over the rapid stress relaxation in later stages (see Fig. 2). It should be noted that the specific volume of the $\mathrm{Cu}_{6} \mathrm{Sn}_{5}$ is larger $(\sim 0.22 \%)$ than the supersaturated solid solution of $\mathrm{Sn}$ and $\mathrm{Cu}^{14}$ and hence formation of $\mathrm{Cu}_{6} \mathrm{Sn}_{5}$ at the Sn grain boundaries would generate compressive stress in the adjacent Sn grains. ${ }^{18}$ Interestingly, the build-up of compressive stress in Sn eventually exhibited saturation after prolonged aging ( $>400 \mathrm{~h}$ ). This saturation in stress may result from the formation of a sufficient number of whiskers in the Sn coating such that the incremental increase in the compressive stress due to IMC formation undergoes quick relaxation due to the incremental growth of whiskers on the surface of the Sn coating. It should be noted that the growth rate of the IMC decreases with time, $t$ (as $h_{\mathrm{IMC}} \sim t^{\mathrm{n}}$, where $n<1$, e.g., 0.5 ). This is also reflected in the decreased growth rate of whiskers in the Sn coatings over time. ${ }^{41}$ This balance between stress generation due to formation of the IMC and the stress relaxation due to whisker growth seems to manifest as a steady-state compressive stress in Sn after prolonged aging.

We would like to emphasize here that, the stress state in an Sn coating at any time will be a complex function of stress relaxation (due to whisker growth, power-law creep, recovery processes etc.) and stress build-up (due to IMC growth and constraints placed on Sn coating by the oxide layer on the free surface and IMC at the Sn-substrate interface). Nevertheless, a saturation in the stress (at the highest compressive value attained during stress evolution) suggests that the IMC growth induced generation of compressive stress in the Sn coating remained dominant compared to stress relaxation (e.g., by creep) during storage of the coating, otherwise the coating should approach a stress-free state. Therefore, stress relaxation in Sn due to power-law creep has only a limited role in whisker formation.

It is also important to note that the stress evolution reported here is based on the standard Stoney's analysis wherein the substrate deforms only elastically under the action of stresses present in the film/coating. Now, as the Sn coating develops compressive stress, the substrate will be under tensile stress. This is to ensure the force balance as the externally applied stress is zero. However, since the substrate is much thicker than the coating $\left(t_{\mathrm{Sn}} /\right.$ $t_{\text {brass }}=60$ in this work) the magnitude of tensile stresses induced in the brass substrate will be negligible. Therefore, tensile stresses in the substrate can safely be ignored. However, when the thickness of the substrate $(\mathrm{Cu} / \mathrm{brass})$ becomes comparable to the thickness of the Sn coating, the tensile stresses in the $\mathrm{Cu} /$ brass can no longer be neglected. In such a scenario, the experimentally measured stress evolution curve becomes the superimposition of stress evolution in the $\mathrm{Cu}$ and $\mathrm{Sn}$ layers. ${ }^{16}$ Furthermore, when Sn is deposited on a $\mathrm{Cu} /$ brass substrate, a scallop-like layer of $\mathrm{Cu}_{6} \mathrm{Sn}_{5}$ IMC forms with time at the coating-substrate interface due to the diffusion reaction between $\mathrm{Cu}$ and Sn. Therefore, the deposited two-layer system of $\mathrm{Sn} /$ brass transforms into a tri-layer $\mathrm{Sn} / \mathrm{Cu}_{6} \mathrm{Sn}_{5} /$ brass system with aging. Lee and Lee ${ }^{10}$ suggested that the measured curvature will, therefore, be proportional to $\sigma_{\mathrm{Sn}} \cdot t_{\mathrm{Sn}}+\sigma_{\mathrm{IMC}} \cdot t_{\mathrm{IMC}}$, where $\sigma_{\mathrm{Sn}}$ and $\sigma_{\text {IMC }}$ are the stresses in Sn coating and IMC layer, respectively, and $t_{\mathrm{Sn}}$ and $t_{\mathrm{IMC}}$ are the thicknesses of the Sn coating and IMC layer, respectively. However, it is important to note that the interfacial IMC layer is not uniform and continuous. Experimental evidence suggests that IMC scallops grow only along grain boundaries and triple points and coarsen with aging to form a continuous network IMC along the grain boundaries after prolonged periods. The effect of such a discontinuous layer on the curvature and hence stress in a film was first modeled by Beuth ${ }^{34}$ by assuming multiple cracks extending through the thickness of the film. Later, Hutchinson developed a modified Stoney's equation by incorporating a factor, called Hutchinson factor, that depends on the ratio of spacing between adjacent cracks, $d$, (which will be the distance between IMC scallops) and layer thickness, $t_{\mathrm{f}}$, (which will be equal to the height of IMC scallops in this case). ${ }^{35}$ When $d \gg t_{\mathrm{f}}$, the Hutchinson factor, $H$, tends to 0 and the normal Stoney's equation is recovered; however, when $d \ll t_{\mathrm{f}}, H$ becomes equal to 1 and the induced curvature is small and can be neglected, leading to a stress-free film. ${ }^{35}$ The average distance between IMC scallops (i.e., $d$ ) and the height of IMC scallops (i.e., $t_{\mathrm{f}}$ ) was measured using SEM image analysis of the substrate after selectively etching the Sn coating, and $H$ was found to be close to 1 . Therefore, according to the aforementioned analysis, the curvature due to a discontinuous IMC layer could be assumed to be negligible, and hence the stress evolution shown in Fig. 2 can be directly attributed solely to the evolution of stress in the Sn coating. The good match between the saturation stress (i.e., - $12 \mathrm{MPa}$ ) observed in this study and that reported previously ${ }^{18-20}$ (using same curvature-based stress measurement method but involving stripping off Sn layer from substrate) validates the continuous real-time stress measurement reported in this work (without removing the Sn coating from the substrate).

Interestingly, as shown in Fig. $4 \mathrm{~b}$, the stress measured via XRD showed the opposite trend in the stress evolution, as the initial stress in the Sn coating was compressive and the stress became less compressive (or, more tensile) with aging. It should be noted that stress measured using XRD was the 
average stress in only the top $0.7 \mu \mathrm{m}$ layer of the $\mathrm{Sn}$ coating. It is now known that under ambient conditions, $\mathrm{Sn}$ forms a stable oxide $\mathrm{SnO}_{2}$. Since $\mathrm{SnO}_{2}$ has a higher molar volume than $\mathrm{Sn},{ }^{42}$ formation of $\mathrm{SnO}_{2}$ along $\mathrm{Sn}$ grain boundaries terminating on the free surface, where diffusion of oxygen is faster, ${ }^{42}$ will induce compressive stress near the surface of the Sn coating. Since the oxide layer is very thin, the compressive stresses will only be induced in the topmost regions. Now, the decrease in the compressive stress in the top layer with time can be explained as follows: (1) disruption of the $\mathrm{SnO}_{2}$ layer along grain boundaries of $\mathrm{Sn}$ due to nucleation and growth of whiskers, and (2) replacement of $\mathrm{SnO}_{2}$ by more stable $\mathrm{ZnO}$. $\mathrm{ZnO}$ forms due to the fast diffusion of $\mathrm{Zn}$ atoms from the brass substrate along the grain boundaries of $\mathrm{Sn}$ and the subsequent reaction with $\mathrm{SnO}_{2}$. The fast diffusion of $\mathrm{Zn}$ was confirmed using electron probe microanalysis (EPMA) in one of our previous studies ${ }^{41}$ and also in a few other studies, ${ }^{43,44}$ where a considerable $\mathrm{Zn}$ concentration was observed near the free surface of the Sn coating. The molar volume of $\mathrm{ZnO}$ is $14.5 \mathrm{~cm}^{3} / \mathrm{mol}^{45}$ which is considerably less than the molar volume of $\mathrm{SnO}_{2}$, which is $21.7 \mathrm{~cm}^{3} / \mathrm{mol}^{42}$ Therefore, replacing $\mathrm{SnO}_{2}$ with $\mathrm{ZnO}$ will relax the existing compressive stress near the surface of the coatings. In summary, formation of $\mathrm{ZnO}$ through the reaction between $\mathrm{SnO}_{2}$ and $\mathrm{Zn}$ and disruption of the oxide layer explains the decrease in the compressive stress near the surface of the Sn coatings with time. Therefore, it can be inferred that the mechanisms of stress generation and stress relaxation at the coating-substrate interface and at the free surface in the Sn coating, respectively, are fundamentally different.

Figure 10 compares the evolution of the bulk stress and the surface stress in Sn coatings, which are individually shown in Figs. 2 and $4 \mathrm{~b}$, respectively. It can be readily observed from Fig. 10 that the surface stress became less compressive relative to the bulk with time and, on average, it became more tensile than the bulk stress after $420 \mathrm{~h}$ ( $\sim 17$ days), as marked by the solid vertical line. Therefore, while it is expected that Sn atoms would tend to migrate from the surface of the coating towards the coating-substrate interface at the beginning of the aging process, Sn atoms would eventually be transported upward towards the surface, thereby supplying the mass flux at the root of the whisker required for its growth. It should be noted that the broken vertical line drawn in Fig. 10 marks the instant that whisker growth was first observed (i.e., after $\sim 180 \mathrm{~h}$ ), and thereby confirms the existence of an incubation period during which stresses and stress gradients increase in the Sn coating. Although the surface is (on an average) more compressive than the bulk overall when the first set of whiskers emerge, the broken oxide layer along the specific grain boundaries of Sn near the surface can provide a stress-free location for diffusion of Sn atoms. A strong directional negative stress gradient towards such a stress-free region would be locally established as soon as a small patch of the oxide breaks, and Sn atoms can be transported towards this region for whisker growth. Because the stress measurement techniques employed are not sensitive to the local stress field and reflect only the average stress, the growth of whiskers due to a local variation in the stress field could not be captured in this study. However, it can be anticipated that as soon as the bulk becomes more compressive on average than the surface, a significant mass flux of Sn atoms would occur from the bulk to the surface, thereby accelerating the growth of whiskers and enabling spontaneous whisker growth over a long period of time. Following an accelerated period of whisker growth, the whisker growth rate would decrease once the buildup of compressive stress in the bulk of Sn due to IMC growth gets saturated. Therefore, by observing stress profiles in the bulk and in the region in the vicinity of the surface of the coating, as shown in Fig. 10, the existence of an incubation period for whisker growth, followed by a period of accelerated whisker growth and finally a decrease in whisker growth can be explained.

In addition, the evolution of bulk stress and surface stress systematically captured here can explain several widely reported observations related to whisker growth in Sn coatings: (1) the evolution of stress and the stress gradient between the surface and the bulk can explain the different incubation periods observed in the previous studies ${ }^{15,46}$ for different Sn coatings, (2) the existence of a positive stress gradient upon prolonged aging favoring transport of atoms from the surface

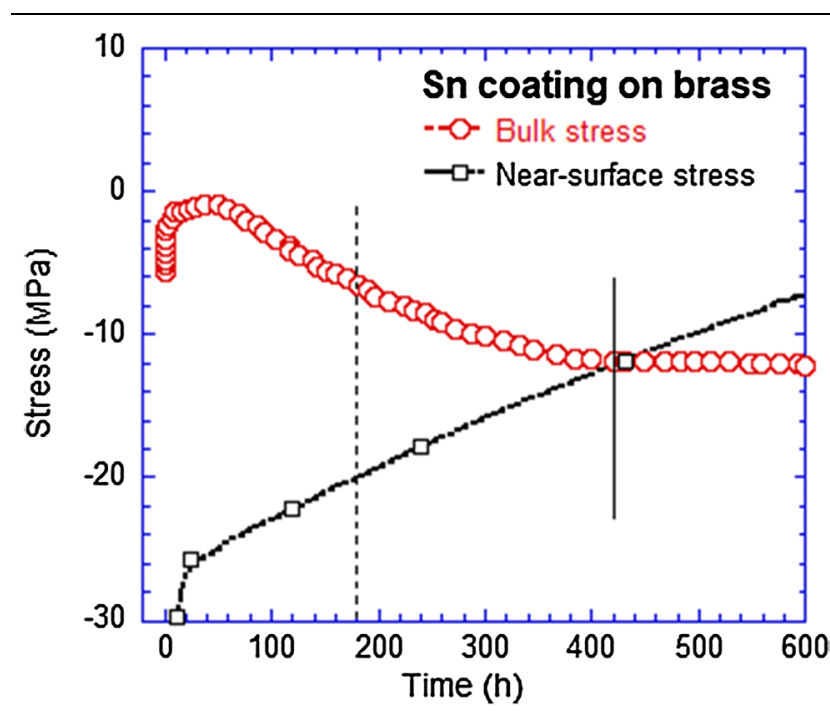

Fig. 10. The evolution of the bulk stress with time measured by the substrate curvature technique and the near-surface stress measured using glancing angle XRD in Sn coating on a brass substrate. The broken and solid vertical lines denote the onset of the first set of whiskers and the instant the bulk became on average more compressive than the surface. 
towards the interface can explain depletion zones or subsiding grains reported in the literature,${ }^{30,47}$ and (3) cross-over of bulk stress and surface stress producing negative stress gradients favoring mass transport of atoms from compressive regions near the IMC towards the whisker root can explain the continued growth of whiskers over time.

\section{Effect of Externally Imposed Stress and Stress Gradient on Whisker Growth}

As discussed in the previous section, both the nearsurface and the bulk stresses and the produced stress gradients play critical roles in whisker growth. The externally applied stress, as shown in Fig. 1, will also affect the stress state in the top layer of the coating, so that the Sn coating subjected to the outward bending (i.e., convex side) will have the maximum tensile stress at the surface and the inward bent (i.e., concave side) coating will have the maximum compressive stress at the coating surface. Therefore, a negative stress gradient, necessary for whisker growth, was inherently imposed in the Sn coatings that are outward bent, which are under tensile stress. The direction of such a stress gradient will be reversed for the inward bent coatings. Based on discussion presented in the section "Effects of Stress and Stress Gradient on Whisker Growth", this makes the outward bent coatings, i.e., the coatings under tensile stress, more prone to whisker growth.

In addition, it was shown in Figs. 8 and 9 that although the thickness (i.e., growth rate) of the interfacial IMC layer was similar in coatings loaded under tensile and compressive stresses, the volume of IMC formed (i.e., nucleation rate of IMC) in the coatings under tension was significantly greater than that of coatings under compression. The enhanced nucleation of IMC in the Sn coatings loaded under tensile stress can be attributed to the fact that most of the columnar grain boundaries of Sn terminating on the substrate experienced a tensile opening force. This enhances the diffusion of $\mathrm{Cu}$ atoms and subsequent nucleation of IMC as compared to the $\mathrm{Sn}$ coating loaded under compressive stress, wherein most of the boundaries experience a compressive stress (see Fig. 1b for schematic illustration). As soon as the interfacial IMC starts to grow, the negative stress gradient in the outward bent coating will be further augmented by the additional influence of the intrinsically generated compressive stress due to the formation of interfacial IMC along Sn grain boundaries. Interestingly, the externally applied tensile stress in the outward bent coating will also break the (brittle) surface oxide layer at several locations, thus increasing the probable sites of whisker growth as well as adding to the existing outward stress gradient. This is in good agreement with the observation that whiskers were longer and grew at a higher rate in outward bent coatings, which were simultaneously under tensile stress, relative to the inward bent coatings.
It should be noted that a similar observation pertaining to the enhanced whisker growth under tensile stress was also reported by Chen et al. ${ }^{15}$ Crandall et al. ${ }^{29}$ and Cheng et al. ${ }^{30}$ However, explanation for these observations was lacking. This work presents a systematic variation in the whisker density as a function of tensile as well as compressive stress, and, more importantly, also explains the whisker growth under tensile stress by considering the stress gradient. It is apparent from the evidence provided in this study that the negative stress gradient, as opposed to stress, dominantly governs whisker growth in Sn coatings.

Although we observe an important role for the negative out-of-plane stress gradient in this work, it is important to highlight here that we did not measure the in-plane stress gradients in the Sn layer and hence cannot conclusively say that out-ofplane stress gradients are more important than inplane stress gradients. However, there are several experimental reports which use synchrotron x-ray micro-diffraction to measure stress/strain gradients around the whiskering site that show that longrange in-plane stress gradients are not very prominent and unique to whisker grain,. ${ }^{48,49}$ The crystal plasticity simulations based on EBSD grain orientation maps around whisker grain also indicate the in-plane stress gradients are only short-range and not uniquely prominent around whisker grain..$^{50}$ Additionally, Kehrer and Kadereit ${ }^{51}$ showed using radioactive tracer experiments that when an inactive Sn layer was deposited on top of a radioactive Sn layer, the whiskers formed on the top surface of the inactive Sn layer were composed of radioactive Sn. This observation confirms that vertical mass transport is more critical than the lateral mass transport. In light of all the experimental and theoretical evidence, we infer that out-of-plane stress gradients play a more important role in the whisker growth process than the nature of the stress alone.

\section{CONCLUSIONS}

- The stress state in Sn coatings on brass was studied using two techniques, substrate curvature measurement using laser-optics and glancing x-ray diffraction. The curvature technique allowed measurement of average bulk stresses in Sn and its evolution with aging. Similarly, the glancing angle x-ray diffraction allowed measurement of average stress in the top $\sim 0.7 \mu \mathrm{m}$ layer near the free surface of the coating and its evolution with aging.

- The near-surface and bulk stresses in the Sn coating evolved differently with time. The bulk stress in the Sn was compressive immediately after electrodeposition; however, it rapidly relaxed and thereafter progressively became more compressive due to IMC growth until stress 
build-up due to IMC formation was balanced by stress relaxation by whisker formation. This led to saturation in stress values. In contrast, stress in the Sn layer near the top surface of the coating became less compressive (i.e., more tensile) with time.

- Initially, a positive stress gradient (i.e., the coating substrate interface was more tensile than the surface) was observed in the $\mathrm{Sn}$; however, the sign of this gradient reversed after a certain aging period (i.e., $400 \mathrm{~h}$ at room temperature), enabling a dramatic increase in the transport of Sn atoms from the highlycompressed Sn layer near the IMC to the whisker root.

- Whisker density increased monotonically with externally imposed tensile stress and negative stress gradient. The enhanced whisker growth was due to the combined effects of the ease of disrupting the surface oxide layer at multiple locations and a negative stress gradient towards these locations that is inherent to such outward bent (or tensile loaded) Sn coatings.

- The externally imposed tensile stress along with the negative stress gradient accelerated the IMC growth such that the density of IMC observed in Sn coatings under tensile stress with a negative stress gradient was higher than that for coatings under compressive stress with a positive stress gradient.

\section{ACKNOWLEDGEMENTS}

This work was financially supported by the Indian Space Research Organization (ISRO) through the Space Technology Cell at the Indian Institute of Science, Bangalore (Grant \# ISTC 0367). Discussions with Dr. Ramesh Narayan P. of Vikram Sarabhai Space Centre, Indian Space Organisation, Trivandrum are also acknowledged. We also thank Professor Rajeev Ranjan and Dr. Amit Sharma of Indian Institute of Science, Bangalore for their help with XRD.

\section{REFERENCES}

1. H. Leidecker and J. Brusse, Tin whiskers: a history of documented electrical system failures (2006). Online at: http://nepp.nasa.gov/whisker.

2. R.M. Fisher, L.S. Darken, and K.G. Carroll, Acta Metall. Mater. 2, 368 (1954).

3. V.K. Glazunova and N.T. Kudryavtsev, Translated from Zhurnal Prikladnoi Khimii 36, 543 (1963).

4. U. Lindborg, Metall. Trans. A 6, 1581 (1975).

5. C.H. Pitt and R.G. Henning, J. Appl. Phys. 35, 459 (1964).

6. M. Sobiech, M. Wohlschlögel, U. Welzel, E.J. Mittemeijer W. Hügel, A. Seekamp, W. Liu, and G.E. Ice, Appl. Phys. Lett. 94, 221901 (2009).

7. K.N. Tu, Acta Metall. 21, 347 (1973).
8. C. Xu, Y. Zhang, C. Fan, A. Vysotskaya, J. Abys, L. Hopkins, and N.F. Stevie, in Proc. AESF SUR/FIN Conf., Jun. 2001.

9. R. Schetty, N. Brown, A. Egli, J. Heber, and A. Vinckler, in Proc. AESF SUR/FIN Conf., (2001) 1.

10. B.Z. Lee and D.N. Lee, Acta Mater. 46, 3701 (1998).

11. S. Lal and T.D. Moyer, IEEE Trans. Electron. Packag. Manuf. 28, 63 (2005).

12. U. Welzel and E.J. Mittemeijer, in Defect and Diffusion Forum, Trans Tech Publications 264 (2007) 71.

13. M. Sobiech, U. Welzel, E.J. Mittemeijer, W. Hügel, and A. Seekamp, Appl. Phys. Lett. 93, 011906-1-3. (2008).

14. W.J. Boettinger, C.E. Johnson, L.A. Bendersky, K.W. Moon, M.E. Williams, and G.R. Stafford, Acta Mater. 53, 305033 (2005).

15. K. Chen and G.D. Wilcox, Phys. Rev. Lett. 94, 066104 (2005).

16. E. Chason, L. Reinbold, and S. Kumar, in MRS Proc., Cambridge University Press, 851 (2004) NN5171.

17. E. Chason, N. Jadhav, W.L. Chan, L. Reinbold, and K.S. Kumar, Appl. Phys. Lett. 92, 171901 (2008).

18. E. Chason, N. Jadhav, and F. Pei, JOM 63, 62 (2011).

19. N. Jadhav, J. Wasserman, F. Pei, and E. Chason, J. Electron. Mater. 41, 588 (2012).

20. E. Chason and F. Pei, JOM 67, 2416 (2015).

21. K.N. Tu and J.C. Li, Mater. Sci. Eng. A 409, 131 (2005).

22. P. Su, M. Ding, and S. Chopin, in Proc. Electron. Comp. And Tech. Conf., (ECTC) (2005) 434-440.

23. K.N. Tu, C. Chen, and A.T. Wu, J. Mater. Sci. Mater. Electron. 18, 269 (2007).

24. J. Liang, Z.H. Xu, and X. Li, J. Mater. Sci. Mater. Electron. 18,599 (2007).

25. V.K. Glazunova, Transl. Kristallografiya 7, 761 (1962).

26. B.D. Dunn, W.R. Burke, and B. Battrick, A laboratory study of tin whisker growth, European Space Agency (ESA) Rep. STR-223 (1987).

27. C. Xu, Y. Zhang, C. Fan, J. Abys, L. Hopkins, and F. Stevie, Circuit Tree (USA) 15, 10 (2002).

28. S.K. Lin, Y. Yorikado, J. Jiang, K.S. Kim, K. Suganuma, S.W. Chen, M. Tsujimoto, and I. Yanada, J. Mater. Res. 22, 1975 (2007)

29. E.R. Crandall, G.T. Flowers, R. Jackson, P. Lall, and M.J. Bozack, in IEEE 57th Conference on Electrical Contacts, Holm (2011) 1.

30. J. Cheng, Tin Whiskers in Electronic Packaging: Mechanism and Modeling, $\mathrm{PhD}$ thesis, University of Rochester (2011) 83.

31. P. Jagtap, Whisker Growth from Electrodeposited Sn Coatings- Developing Materials Science and Mechanics Based Insights, $\mathrm{PhD}$ thesis, Indian Institute of Science (2017).

32. J. A. Floro and E. Chason, in In-Situ Real-Time Characterization of Thin Films, Ed. O. Auciello and A. R. Krauss, John Wiley and Sons, Inc. (2001) 191.

33. V.A. Sethuraman, M.J. Chon, M. Shimshak, V. Srinivasan, and P.R. Guduru, J. Power Sources 195, 5062 (2010).

34. J.L. Beuth, Int. J. Solids Structures 29, 1657 (1992).

35. W.D. Nix, Mechanical properties of thin films, Lecture notes (2005) Available online at: http://imechanica.org/files/ 353ClassNotes2005.pdf

36. U. Welzel, J. Ligot, P. Lamparter, A.C. Vermeulen, and E.J. Mittemeijer, J. Appl. Crystallogr. 38, 1 (2005).

37. A. Kumar, U. Welzel, and E.J. Mittemeijer, J. Appl. Crystallogr. 39, 633 (2006).

38. B. D. Cullity, Elements of x-ray Diffraction, Add1sonWesley Publishing Company, Inc. (1956) 466.

39. Y.C. Cheng, Y.T. Wang, F.C. Hsu, F.C. Lu, C.L. Wu, and M.T. Lin, J. Electron. Mater. 44, 604 (2015).

40. W.F. Gale and T.C. Totemeier, eds., Smithells metals reference book (Oxford: Butterworth-Heinemann, 2003), p. 1373.

41. P. Jagtap and P. Kumar, J. Electron. Mater. 44, 1206 (2015). 
42. J.W. Osenbach, J.M. DeLucca, B.D. Potteiger, A. Amin, R.L. Shook, and F.A. Baiocchi, IEEE Trans. Electron. Packag. Manuf. 30, 23 (2007).

43. M.A. Ashworth, G.D. Wilcox, R.L. Higginson, R.J. Heath, and C. Liu, J. Electron. Mater. 43, 1005 (2014).

44. S.C. Britton and M. Clarke, Trans. Inst. Metal Finish. 40, 205 (1964).

45. F. Han, W.C. Li, C. Lei, B. He, K. Oshida, and A.H. Lu, Small 10, 2637 (2014).

46. E. Bradley, C.A. Handwerker, J. Bath, R.D. Parker, and R.W. Gedney, Lead-free electronics: iNEMI projects lead to successful manufacturing, John Wiley \& Sons (2007) 305.
47. Y. Wang, J.E. Blendell, and C.A. Handwerker, J. Mater. Sci. 49, 1099 (2014).

48. W.J. Choi, T.Y. Lee, K.N. Tu, N. Tamura, R.S. Celestre, A.A. MacDowell, Y.Y. Bong, and L. Nguyen, Acta Mater. 51,6253 (2003).

49. F. Pei, N. Jadhav, E. Buchovecky, A.F. Bower, E. Chason, W. Liu, J.Z. Tischler, G.E. Ice, and R. Xu, J. Appl. Phys. $119,105302(2016)$.

50. P. Jagtap, A. Chakraborty, P. Eisenlohr, and P. Kumar, Acta Mater. 134, 346 (2017).

51. H.P. Kehrer and H.G. Kadereit, Appl. Phys. Lett. 16, 411 (1970). 